\title{
Transmission of vibration through glove materials: effects of contact force
}

\begin{abstract}
This study investigated effects of applied force on the apparent mass of the hand, the dynamic stiffness of glove materials, and the transmission of vibration to the hand. For 10 subjects, three glove materials, and three contact forces, apparent masses and glove transmissibilities were measured at the palm and at a finger at frequencies in the range 5 to $300 \mathrm{~Hz}$. The dynamic stiffnesses of the materials were also measured. With increasing force, the dynamic stiffnesses of the materials increased, the apparent mass at the palm increased at frequencies greater than the resonance, and the apparent mass at the finger increased at low frequencies. The effects of force on transmissibilities therefore differed between materials and depended on vibration frequency, but changes in apparent mass and dynamic stiffness had predictable effects on material transmissibility. Depending on the glove material, the transmission of vibration through a glove can be increased or decreased when increasing the applied force.
\end{abstract}

Keyword: Anti-vibration gloves; Biodynamics; Transmissibility; Impedance; Hands; Fingers 